\title{
An Extended Target CPHD Filter and a Gamma Gaussian Inverse Wishart Implementation
}

\author{
Christian Lundquist, Karl Granström and Umut Orguner
}

\section{Linköping University Post Print}

\section{Tweet}

N.B.: When citing this work, cite the original article.

(C2013 IEEE. Personal use of this material is permitted. However, permission to reprint/republish this material for advertising or promotional purposes or for creating new collective works for resale or redistribution to servers or lists, or to reuse any copyrighted component of this work in other works must be obtained from the IEEE.

Christian Lundquist, Karl Granström and Umut Orguner, An Extended Target CPHD Filter and a Gamma Gaussian Inverse Wishart Implementation, 2013, IEEE Journal on Selected Topics in Signal Processing, (7), 3, 472-483.

http://dx.doi.org/10.1109/JSTSP.2013.2245632

Postprint available at: Linköping University Electronic Press

http://urn.kb.se/resolve?urn=urn:nbn:se:liu:diva-94596 


\title{
An Extended Target CPHD Filter and a Gamma Gaussian Inverse Wishart Implementation
}

\author{
Christian Lundquist, Karl Granström, Member, IEEE, and Umut Orguner, Member, IEEE
}

\begin{abstract}
This paper presents a cardinalized probability hypothesis density (CPHD) filter for extended targets that can result in multiple measurements at each scan. The probability hypothesis density (PHD) filter for such targets has been derived by Mahler, and different implementations have been proposed recently. To achieve better estimation performance this work relaxes the Poisson assumptions of the extended target PHD filter in target and measurement numbers. A gamma Gaussian inverse Wishart mixture implementation, which is capable of estimating the target extents and measurement rates as well as the kinematic state of the target, is proposed, and it is compared to its PHD counterpart in a simulation study. The results clearly show that the CPHD filter has a more robust cardinality estimate leading to smaller OSPA errors, which confirms that the extended target CPHD filter inherits the properties of its point target counterpart.
\end{abstract}

Index Terms-Multiple target tracking, extended targets, random sets, probability hypothesis density, PHD, cardinalized, CPHD, random matrices, inverse Wishart.

\section{INTRODUCTION}

$\mathbf{M}$ ULTIPLE TARGET TRACKING can be defined as the processing of multiple measurements obtained from multiple targets in order to maintain estimates of the targets' current states, see e.g., [1]. In this context, a point target is defined as a target which is assumed to give rise to at most one measurement per time step, and an extended target is defined as a target that potentially gives rise to more than one measurement per time step. Closely related to extended target is group target, defined as a cluster of point targets which can not be tracked individually, but has to be treated as a single object which can give rise to multiple measurements.

The point target assumption is valid for some cases, e.g., in radar based air surveillance applications when the distance between the target and the sensor is large. However, in other cases the resolution of the sensor, the size of the target, or the distance between target and sensor, might be such that multiple resolution cells of the sensor are occupied by the target. Examples of such extended target scenarios include vehicle tracking using automotive radar, tracking of sufficiently close airplanes or ships with ground or marine radar stations, and person tracking using laser range sensors.

Copyright (c) 2013 IEEE. Personal use of this material is permitted. However, permission to use this material for any other purposes must be obtained from the IEEE by sending a request to pubs-permissions@ieee.org

C. Lundquist and K. Granström are with the Division of Automatic Control, Department of Electrical Engineering, Linköping University, SE-581 83 Linköping, Sweden, e-mail: \{lundquist, karl\}@isy. liu.se.

U. Orguner is with Department of Electrical \& Electronics Engineering, Middle East Technical University, 06531 Ankara, Turkey, e-mail: umut@metu.edu.tr
With a possibility of multiple measurements per target, a model for the number of measurements per target is needed. Gilholm and Salmond [2] presented an approach for tracking extended targets under the assumption that the number of received target measurements in each time step is Poisson distributed. In [3] a measurement model was suggested which is an inhomogeneous Poisson point process. At each time step, a Poisson distributed random number of measurements are generated, distributed around the target. This measurement model can be understood to imply that the extended target is sufficiently far away from the sensor, in relation to the sensor's resolution, for its measurements to resemble a cluster of points, rather than a geometrically structured ensemble.

Multiple measurements per target also raise the possibility of estimating the extended target's extension, i.e., the size and shape of the target. Using random matrices as a model for extended targets and groups of targets was suggested by Koch in 2008 [4]. The target kinematical states are modeled as Gaussian distributed, while the target extension is modeled as inverse Wishart distributed. Using random matrices to track group targets under kinematical constraints is discussed in [5]. Modifications and improvements to the Gaussian inverse Wishart model of [4] have been suggested in [6], and the model [4] has also been integrated into a Probabilistic Multi-Hypothesis Tracking (РMHT) framework in [7]. Further approaches to estimating the target extensions, as ellipses, rectangles, or more general shapes, are given in e.g., [8]-[13].

With finite set statistics (FISST), Mahler introduced a set theoretic approach in which targets and measurements are modeled using random finite sets (RFS). The approach allows multiple target tracking in the presence of clutter and with uncertain associations to be cast in a Bayesian framework [14], resulting in an optimal multi-target Bayes filter. An important contribution of FISST is the statistical moments of the RFS, which enable practical implementation of the optimal multitarget Bayes filter. The first order moment of an RFS is called the probability hypothesis density (PHD), and it is an intensity function defined over the target state space. The PHD filter propagates the target set's PHD in time [14], [15], and represents an approximation to the optimal multi-target Bayes filter.

By approximating the PHD with a Gaussian mixture (GM), a practical implementation of the PHD filter for point targets is obtained, called the Gaussian mixture PHD (GM-PHD) filter [16]. Convergence analysis of the GM-PHD filter is given in [17]. A sequential Monte Carlo implementation of the point target PHD filter is given in [18], with convergence analysis in [18]-[20]. 
An extension of the PHD filter to handle extended targets of the type presented in [3] is given in [21]. A Gaussian mixture implementation of the extended target PHD filter [21], called the ET-GM-PHD-filter, has been presented in [22], with an early version given in [23]. In both of the works [22] and [23], only the kinematic properties of the targets' centroids are estimated. Estimating the targets' extensions is omitted to reduce the complexity of the presentation. An implementation of [21] that utilizes the random matrix based extended target model [4] was presented in [24], the resulting filter is called the Gaussian inverse Wishart PHD filter (GIW-PHD filter).

An extended target PHD filter is given in [25], derived under slightly different model assumptions than those in [21]. A Gaussian mixture implementation is given, however the proposed implementation does not consider approximation of the full set of measurement set partitions, which is shown in [22]-[24] to be of great importance. A Gaussian Mixture Markov Chain Monte Carlo filter for multiple extended target tracking is presented in [26]. The filter is compared to the linear ET-GM-PHD-filter [22], [23], and is shown to be less sensitive to clutter (number of cardinality errors: 133 vs. 373 ) but also considerably more computationally demanding (mean cycle time: $3.23 \mathrm{~s}$ vs. $0.14 \mathrm{~s})$.

For the closely related area of group target tracking, in which several targets move in unison, an approach using the point target GM-PHD filter is presented in [27]. An RFS formulation of single extended target tracking is given in [28].

A known drawback of the PHD filter is that the cardinality is estimated using a single parameter (the mean), resulting from the cardinality distribution being approximated with a Poisson distribution. Because the mean and the variance of a Poisson density are equal, when the true cardinality is high the corresponding estimate has a high variance. In practice, this results in an oversensitive cardinality estimate, e.g., seen when there are missed detections [29]. To improve upon this, the cardinalized probability hypothesis density (CPHD) filter was introduced [30]. In addition to propagating the PHD in time, the CPHD filter also propagates the full cardinality distribution. A GM implementation of the CPHD filter for point targets is given in [31].

The first steps towards a CPHD filter for extended targets appeared in [32], however no implementation was proposed. While only the PHD updates are given in [32], the cardinality updates can be extracted from the given equations. The models used in [32] were later used to derive a PHD filter in [25]. A CPHD filter for extended targets was presented in [33], however the filter derivation is based on the quite strong assumption that "relative to sensor resolution, the extended targets and the unresolved targets are not too close and the clutter density is not too large" [33, Corollary 1]. This assumption cannot be expected to hold in the general case.

This paper presents a CPHD filter for extended targets and group targets, denoted ET-CPHD. The ET-CPHD filter is derived under a different model than that used in [32], and thus the resulting equations are not the same. An early version of this work was presented in [34], [35], where a GM implementation was shown.

In this paper we just describe the results of the derivation of the ET-CPHD filter as in [34] and then give a gamma Gaussian inverse Wishart (GGIW) implementation of it. The implementation can easily handle Bayesian estimation of the targets' extensions and measurement rates, as well as the kinematic states of the targets. The GGIW implementation can be reduced to the GM filter presented in [34] with the additional measurement error covariance estimation capability thanks to the properties of the random matrix framework [4]. The filter derivation does not require any assumptions regarding the spatial proximity of the targets, or the clutter density. Indeed, it is shown in the results section that the presented filter handles both high clutter density and spatially close targets. In this sense the presented extended target CPHD filter is more general than the CPHD filter presented in [33].

The rest of the paper is organized as follows. In Section II we give a problem formulation, and in Section III we give the main update formulae of a CPHD filter for extended targets. Section IV presents the extended target state model, and the implementation of the CPHD filter is given in Section V. Target extraction and performance evaluation metrics are presented in Section VI, and the results from a simulation study are given in Section VII. Section VIII contains conclusions and thoughts on future work.

\section{Problem Formulation}

Let $\xi_{k}^{(i)}$ denote the state of the $i$ :th extended target at time $k$, and let the set of extended targets at time $k$ be denoted

$$
\mathbf{X}_{k}=\left\{\xi_{k}^{(i)}\right\}_{i=1}^{N_{\xi, k}}
$$

where $N_{\xi, k}$ is the unknown, time-varying, number of extended targets. Let the operation $|\cdot|$ denote set cardinality, i.e., $\left|\mathbf{X}_{k}\right|=$ $N_{\xi, k}$. The set of target generated measurements obtained at time $k$ is denoted

$$
\mathbf{Z}_{\mathrm{T}, k}=\left\{\mathbf{z}_{k}^{(j)}\right\}_{j=1}^{N_{\mathrm{T}, k}}
$$

where $N_{\mathrm{T}, k}=\left|\mathbf{Z}_{\mathrm{T}, k}\right|$ is the number of measurements. The set of target measurements is distributed according to an i.i.d. cluster process. The corresponding set likelihood is given as

$$
f\left(\mathbf{Z}_{\mathrm{T}, k} \mid \xi\right)=N_{\mathrm{T}, k} ! P_{\mathbf{z}}\left(N_{\mathrm{T}, k} \mid \xi\right) \prod_{\mathbf{z}_{k} \in \mathbf{Z}_{\mathrm{T}, k}} p_{\mathbf{z}}\left(\mathbf{z}_{k} \mid \xi\right)
$$

where $P_{\mathbf{z}}(\cdot \mid \xi)$ and $p_{\mathbf{z}}(\cdot \mid \xi)$ denote the probability mass function (pmf) for the cardinality $N_{\mathrm{T}, k}$ of the measurement set $\mathbf{Z}_{\mathrm{T}, k}$, and the likelihood of a single measurement, conditioned on the state $\xi$ of the target. Note here our convention of showing the dimensionless probabilities with " $P$ " and the likelihoods with " $p$ ".

The set of false alarms collected at time $k$ is denoted

$$
\mathbf{Z}_{\mathrm{FA}, k}=\left\{\mathbf{z}_{k}^{(j)}\right\}_{j=1}^{N_{\mathrm{FA}, k}}
$$

The false alarms are distributed according to an i.i.d. cluster process with set likelihood

$$
f\left(\mathbf{Z}_{\mathrm{FA}, k}\right)=N_{\mathrm{FA}, k} ! P_{\mathrm{FA}}\left(N_{\mathrm{FA}, k}\right) \prod_{\mathbf{z}_{k} \in \mathbf{Z}_{\mathrm{FA}, k}} p_{\mathrm{FA}}\left(\mathbf{z}_{k}\right),
$$




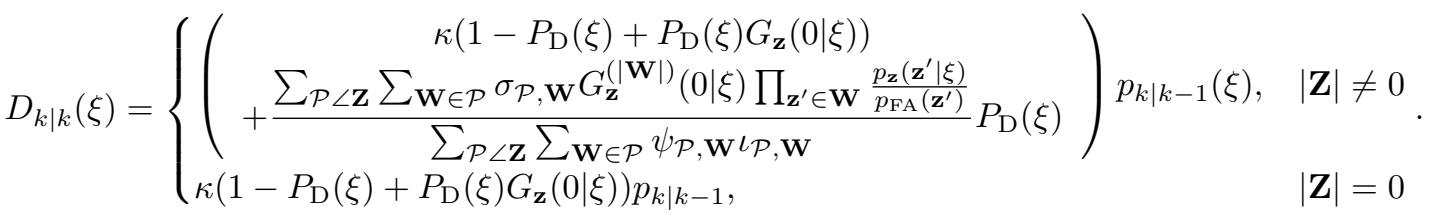

$$
\begin{aligned}
& P_{k \mid k}(n)= \begin{cases}\frac{\sum_{\mathcal{P} \angle \mathbf{Z}} \sum_{\mathbf{W} \in \mathcal{P}} \psi_{\mathcal{P}, \mathbf{W}} G_{k \mid k-1}^{(n)}(0)\left(\begin{array}{c}
G_{\mathrm{FA}}(0) \frac{\eta \mathbf{W}}{|\mathcal{P}|} \frac{\rho^{n-|\mathcal{P}|}}{(n-|\mathcal{P}|) !} \delta_{n \geq|\mathcal{P}|} \\
+G_{\mathrm{FA}}^{(|\mathbf{W}|)}(0) \frac{\rho^{n-|\mathcal{P}|+1}}{(n-|\mathcal{P}|+1) !} \delta_{n \geq|\mathcal{P}|-1}
\end{array}\right)}{\sum_{\mathcal{P}<\mathbf{Z}} \sum_{\mathbf{W} \in \mathcal{P}} \psi_{\mathcal{P}, \mathbf{W} \iota \mathcal{P}, \mathbf{W}}}, & |\mathbf{Z}| \neq 0 . \\
\frac{\rho^{n} G_{k \mid k-1}^{(n)}(0)}{G_{k \mid k-1}(\rho)}, & |\mathbf{Z}|=0\end{cases}
\end{aligned}
$$

where $P_{\mathrm{FA}}(\cdot)$ and $p_{\mathrm{FA}}(\cdot)$ denote the pmf for the cardinality $N_{\mathrm{FA}, k}$ of the false alarm set $\mathbf{Z}_{\mathrm{FA}, k}$, and the likelihood of a single false alarm.

Let $\mathbf{Z}_{k}$ denote the union of the target generated measurements and the false alarms,

$$
\mathbf{Z}_{k}=\left\{\mathbf{z}_{k}^{(j)}\right\}_{j=1}^{N_{\mathbf{z}, k}}=\mathbf{Z}_{\mathrm{T}, k} \cup \mathbf{Z}_{\mathrm{FA}, k},
$$

and let $\mathbf{Z}^{k}$ denote the set of all measurement set, from time 0 to time $k$,

$$
\mathbf{Z}^{k}=\left\{\mathbf{Z}_{0}, \ldots, \mathbf{Z}_{k}\right\} .
$$

The multi-target prior $f\left(\mathbf{X}_{k} \mid \mathbf{Z}^{k-1}\right)$ at each estimation step is assumed to be an i.i.d. cluster process,

$$
f\left(\mathbf{X}_{k} \mid \mathbf{Z}^{k-1}\right)=n ! P_{k \mid k-1}(n) \prod_{\xi \in \mathbf{X}_{k}} p_{k \mid k-1}(\xi),
$$

where $P_{k \mid k-1}(n)$ is the pmf for the cardinality of the set of targets. The predicted single target density is

$$
p_{k \mid k-1}(\xi) \triangleq N_{k \mid k-1}^{-1} D_{k \mid k-1}(\xi)
$$

where $N_{k \mid k-1} \triangleq \int D_{k \mid k-1}(\xi) \mathrm{d} \xi$ and $D_{k \mid k-1}(\xi)$ is the predicted PHD.

Given the above, the aim of the next section will be to derive the CPHD filter equations for extended targets, i.e., to find the posterior PHD $D_{k \mid k}(\xi)$ and the posterior cardinality distribution $P_{k \mid k}(n)$ of the target set $\mathbf{X}_{k}$ given the set of measurements $\mathbf{Z}^{k}$.

\section{CPHD FILTER FOR EXTENDED TARGETS}

The CPHD filter propagates the PHD $D_{k \mid k}(\xi)$ and the cardinality distribution $P_{k \mid k}(n)$. Note that the ET-CPHD predictor equations are equivalent to the standard CPHD predictor equations in [30], and they are omitted for space considerations. In Section III-A the ET-CPHD corrector equations are given without any derivation in view of the fact that the derivations are already given in [34], [35]. Similar equations, derived under different model assumptions, can be found in [32]. Section III-B gives a brief comparison between the ET-CPHD model and the model used in [32].

\section{A. CPHD correction}

The posterior PHD $D_{k \mid k}(\xi)$ and the posterior cardinality distribution $P_{k \mid k}(n)$ of the target set $\mathbf{X}_{k}$ are given in (10) and (11) at the top of the page. The following notation is used in (10) and (11):

- The functions $G_{k \mid k-1}(\cdot), G_{\mathrm{FA}}(\cdot)$ and $G_{\mathbf{z}}(\cdot \mid \xi)$ denote the predicted probability generating function of the state, the probability generating function of the false alarms, and the probability generating function of the measurements conditioned on the state, respectively.

- The superscript $(n)$, indicates the $n$ :th derivative of the corresponding function.

- $\mathcal{P} \angle \mathbf{Z}$ denotes that $\mathcal{P}$ partitions the measurement set $\mathbf{Z}$ into non-empty subsets. When used under a summation sign the summation is over all possible partitions $\mathcal{P}$.

- $|\mathcal{P}|$ denotes the number of non-empty subsets in the partition $\mathcal{P}$.

- The non-empty subsets are denoted $\mathbf{W}$, and are called cells. When $\mathbf{W} \in \mathcal{P}$ is used under a summation sign, the summation is over all cells in the partition.

- $|\mathbf{W}|$ denotes the number of measurements in the cell (i.e., the cardinality).

The coefficients and constants that are utilized in (10) and (11) are defined as follows.

$$
\begin{gathered}
\rho \triangleq p_{k \mid k-1}\left[1-P_{\mathrm{D}}(\cdot)+P_{\mathrm{D}}(\cdot) G_{\mathbf{z}}(0 \mid \cdot)\right], \\
\eta_{\mathbf{W}} \triangleq p_{k \mid k-1}\left[P_{\mathrm{D}}(\cdot) G_{\mathbf{z}}^{(|\mathbf{W}|)}(0 \mid \cdot) \prod_{\mathbf{z}^{\prime} \in \mathbf{W}} \frac{p_{\mathbf{z}}\left(\mathbf{z}^{\prime} \mid \cdot\right)}{p_{\mathrm{FA}}\left(\mathbf{z}^{\prime}\right)}\right], \\
\iota_{\mathcal{P}, \mathbf{W}} \triangleq G_{\mathrm{FA}}(0) G_{k \mid k-1}^{(|\mathcal{P}|)}(\rho) \frac{\eta_{\mathbf{W}}}{|\mathcal{P}|}+G_{\mathrm{FA}}^{(|\mathbf{W}|)}(0) G_{k \mid k-1}^{(|\mathcal{P}|-1)}(\rho),
\end{gathered}
$$

$\chi_{\mathcal{P}, \mathbf{W}} \triangleq G_{\mathrm{FA}}(0) G_{k \mid k-1}^{(|\mathcal{P}|+1)}(\rho) \frac{\eta_{\mathbf{W}}}{|\mathcal{P}|}+G_{\mathrm{FA}}^{(|\mathbf{W}|)}(0) G_{k \mid k-1}^{(|\mathcal{P}|)}(\rho)$,

$\psi_{\mathcal{P}, \mathbf{W}} \triangleq \prod_{\mathbf{W}^{\prime} \in \mathcal{P}-\mathbf{W}} \eta_{\mathbf{W}^{\prime}}$,

$$
\kappa \triangleq\left\{\begin{array}{ll}
\frac{\sum_{\mathcal{P} \angle \mathbf{Z}} \sum_{\mathbf{W} \in \mathcal{P}} \psi_{\mathcal{P}, \mathbf{w}} \chi_{\mathcal{P}, \mathbf{w}}}{\sum_{\mathcal{P} \angle \mathbf{Z}} \sum_{\mathbf{W} \in \mathcal{P}} \psi_{\mathcal{P}, \mathbf{W}} \iota_{\mathcal{P}, \mathbf{W}}}, & |\mathbf{Z}| \neq 0 \\
N_{k \mid k-1}, & |\mathbf{Z}|=0
\end{array},\right.
$$




$$
+\frac{\sum_{\mathbf{W}^{\prime} \in \mathcal{P}-\mathbf{W}} \psi_{\mathcal{P}, \mathbf{W}^{\prime} \iota_{\mathcal{P}, \mathbf{W}^{\prime}}}}{\eta_{\mathbf{W}}} .
$$

where the notation $p[g(\cdot)]$ denotes the integral $\int p(x) g(x) \mathbf{x}$.

\section{B. Comparison to [32]}

The previous work that is most similar to the presented ETCPHD filter is [32]. The PHD correction equation in [32] is derived using hierachical cluster processes, with two levels of cluster processes: the parent and the daughter process, where the daughter process is conditioned on the parent process. In (3), (5) and (8) the target measurement likelihood, false alarm likelihood and multi-target prior are modelled as cluster processes. Thus, the model used here can be seen as a special case of the hierarchical model in [32], where an impulsive likelihood is used for the daughter process.

The increased generality of the hierachical model comes at the price of an additional requirement in the correction formulae: obtaining all sub-partitions of the partitions of the measurement set. In the presented formulation, the correction equations involve only the partitions of the measurement set, i.e., no sub-partitions. This makes the computational complexity of the presented ET-CPHD filter similar to that of the extended target PHD filter [21]. It has been shown in previous work that the measurement set partitioning is of high importance for the practical performance [22]-[24]. Since no implementation is given in [32], a practical comparison is not possible.

\section{The GGIW Extended TARget ModeL}

In this section we present a gamma Gaussian inverse Wishart extended target model.

\section{A. Notation}

We use the following notation:

- $\mathbb{R}^{n}$ is the set of real $n$-vectors, $\mathbb{S}_{++}^{n}$ is the set of symmetric positive definite $n \times n$ matrices, and $\mathbb{S}_{+}^{n}$ is the set of symmetric positive semi-definite $n \times n$ matrices.

- $\mathcal{G} \mathcal{A M}(\gamma ; \alpha, \beta)$ denotes a gamma probability density function (pdf) defined over $\gamma>0$ with shape parameter $\alpha>0$ and inverse scale parameter $\beta>0$,

$$
\mathcal{G} \mathcal{A M}(\gamma ; \alpha, \beta)=\frac{\beta^{\alpha}}{\Gamma(\alpha)} \gamma^{\alpha-1} e^{-\beta \gamma}
$$

- $\mathcal{N}(\mathbf{x} ; m, P)$ denotes a multi-variate Gaussian pdf defined over the vector $\mathbf{x} \in \mathbb{R}^{n_{x}}$ with mean vector $m \in$ $\mathbb{R}^{n_{x}}$, and covariance matrix $P \in \mathbb{S}_{+}^{n_{x}}$,

$$
\mathcal{N}(\mathbf{x} ; m, P)=\frac{e^{-\frac{1}{2}(\mathbf{x}-m)^{\mathrm{T}} P^{-1}(\mathbf{x}-m)}}{(2 \pi)^{\frac{n_{x}}{2}}|P|^{\frac{1}{2}}},
$$

where $|P|$ is determinant of the matrix $P$.

- $\mathcal{I W}_{d}(X ; v, V)$ denotes an inverse Wishart pdf defined over the matrix $X \in \mathbb{S}_{++}^{d}$ with degrees of freedom $v>$ $2 d$ and parameter matrix $V \in \mathbb{S}_{++}^{d}$, [36, Definition 3.4.1]

$\mathcal{I W}_{d}(X ; v, V)=\frac{2^{-\frac{v-d-1}{2}}|V|^{\frac{v-d-1}{2}}}{\Gamma_{d}\left(\frac{v-d-1}{2}\right)|X|^{\frac{v}{2}}} \operatorname{etr}\left(-\frac{1}{2} X^{-1} V\right)$, where $\operatorname{etr}(A)=\exp (\operatorname{Tr}(A))$ is exponential of the trace of the matrix $A$.

- $A \otimes B$ is Kronecker product between matrices $A$ and $B$.

\section{B. Extended Target State}

The extended targets considered in this work are, as described in e.g., [24], characterized by a number of reflection points spread over their extents. The following assumption about the number of measurements generated by each extended target is common in extended target tracking, see e.g., [2], [3], [21]-[24], [37].

Assumption 1: The number of measurements generated by the extended target $\xi_{k}$ is Poisson distributed, with a gamma distributed rate parameter $\gamma_{k}$.

Note that Assumption 1 is generally not needed for implementation of the CPHD filter, it is only made for the specific implementation presented in this paper.

The following assumption about the extended target kinematics (i.e., position, velocity, acceleration) and extension (i.e., shape and size) was first suggested by Koch [4]. It has been used extensively since, see e.g., [5]-[7], [24], [38].

Assumption 2: The target kinematics and target extension can be decomposed into a random vector $\mathbf{x}_{k}$ and a random matrix $X_{k}$.

Modeling the extension as a random matrix limits the extended targets to be shaped as ellipses, comments on the appropriateness of this model can be found in e.g., [4], [6], [24].

The following assumption about the measurement rate and the kinematical and extension states is inherited from [37], where it is noted that it is resonable in most cases.

Assumption 3: The measurement rate $\gamma_{k}$ is conditionally independent of $\mathbf{x}_{k}$ and $X_{k}$.

Since, in general, it is unknown how $\gamma_{k}$ changes over time, conditioned on the kinematics and extension ${ }^{1}$, we believe the assumption, which greatly facilitates the derivation of the GGIW-CPHD filter below, is approximately valid in many cases.

Following Assumptions 1, 2 and 3, the extended target state $\xi_{k}$, cf. (1), is defined as the triple

$$
\xi_{k} \triangleq\left(\gamma_{k}, \mathbf{x}_{k}, X_{k}\right)
$$

where $\gamma_{k}>0$ is the measurement rate, $\mathbf{x}_{k} \in \mathbb{R}^{n_{x}}$ is the kinematical state and $X_{k} \in \mathbb{S}_{++}^{d}$ is the extension state. Following [4], [37], the extended target state $\xi_{k}$, conditioned on $\mathbf{Z}^{k}$, is modeled as gamma Gaussian inverse Wishart (GGIW) distributed,

$$
\begin{aligned}
p\left(\xi_{k} \mid \mathbf{Z}^{k}\right)= & p\left(\gamma_{k} \mid \mathbf{Z}^{k}\right) p\left(\mathbf{x}_{k} \mid X_{k}, \mathbf{Z}^{k}\right) p\left(X_{k} \mid \mathbf{Z}^{k}\right) \\
= & \mathcal{G} \mathcal{A} \mathcal{M}\left(\gamma_{k} ; \alpha_{k \mid k}, \beta_{k \mid k}\right) \\
& \times \mathcal{N}\left(\mathbf{x}_{k} ; m_{k \mid k}, P_{k \mid k} \otimes X_{k}\right) \\
& \times \mathcal{I} \mathcal{W}_{d}\left(X_{k} ; v_{k \mid k}, V_{k \mid k}\right) \\
= & \mathcal{G} \mathcal{G} \mathcal{I} \mathcal{W}\left(\xi_{k} ; \zeta_{k \mid k}\right),
\end{aligned}
$$

where $\zeta_{k \mid k}=\left\{\alpha_{k \mid k}, \beta_{k \mid k}, m_{k \mid k}, P_{k \mid k}, v_{k \mid k}, V_{k \mid k}\right\}$ is the set of GGIW density parameters. The Gaussian covariance is $\left(P_{k \mid k} \otimes\right.$

${ }^{1}$ It is unknown, for example, how many more reflections would appear as the target comes closer to the sensor. 
$\left.X_{k}\right) \in \mathbb{S}_{+}^{n_{x}}$, where $P_{k \mid k} \in \mathbb{S}_{+}^{s}$. Estimates of the kinematic state covariance and of the target extent are obtained as in [4],

$$
\hat{P}_{k \mid k}=\frac{P_{k \mid k} \otimes V_{k \mid k}}{v_{k \mid k}+n_{x}\left(\frac{1}{d}-1\right)-2}, \quad \hat{X}_{k \mid k}=\frac{V_{k \mid k}}{v_{k \mid k}-2 d-2}
$$

for $v_{k \mid k}$ such that the denominators are positive.

\section{Transition Density and Measurement Likelihood}

The state transition density $p\left(\xi_{k+1} \mid \xi_{k}\right)$ describes the time evolution of the extended target state from time $t_{k}$ to time $t_{k+1}$. In Bayesian state estimation, the prediction step consists of solving the Chapman-Kolmogorov equation

$$
p\left(\xi_{k+1} \mid \mathbf{Z}^{k}\right)=\int p\left(\xi_{k+1} \mid \xi_{k}\right) p\left(\xi_{k} \mid \mathbf{Z}^{k}\right) \mathrm{d} \xi_{k} .
$$

The following assumption is made about the transition density.

Assumption 4: The extended target state transition density satisfies

$$
\begin{aligned}
p\left(\xi_{k+1} \mid \xi_{k}\right) & \approx p^{\gamma}\left(\gamma_{k+1} \mid \gamma_{k}\right) p^{\mathbf{x}, X}\left(\mathbf{x}_{k+1}, X_{k+1} \mid \mathbf{x}_{k}, X_{k}\right) \\
& \approx p^{\gamma}\left(\gamma_{k+1} \mid \gamma_{k}\right) p^{\mathbf{x}}\left(\mathbf{x}_{k+1} \mid X_{k+1}, \mathbf{x}_{k}\right) p^{X}\left(X_{k+1} \mid X_{k}\right)
\end{aligned}
$$

for all $\xi_{k}, \xi_{k+1}, \mathbf{x}_{k}, X_{k}, \mathbf{x}_{k+1}$ and $X_{k+1}$.

The approximation in (20b) is inherited from [4], where it is noted that it implies restrictions that can be justified in many practical cases. The approximation to predict the measurement rate independent of the kinematical state and extension state is inherited from [37]. Comments on the pros and cons of this approximation can be found in [37].

The individual measurement likelihood $p_{\mathbf{z}}\left(\mathbf{z}_{k}^{(j)} \mid \xi_{k}\right)$ in (3) describes the relation between the measurements $\mathbf{z}_{k}^{(j)} \in \mathbf{Z}_{k}$ generated by a target and the corresponding target state $\xi_{k}$. The following assumption is made about the measurement likelihood.

Assumption 5: The individual measurement likelihood is given as

$$
p_{\mathbf{z}}\left(\mathbf{z}_{k}^{(j)} \mid \xi_{k}\right)=p_{\mathbf{z}}\left(\mathbf{z}_{k}^{(j)} \mid \mathbf{x}_{k}, X_{k}\right)
$$

Note that the proposed likelihood $p_{\mathbf{z}}\left(\mathbf{z}_{k}^{(j)} \mid \xi_{k}\right)$ does not depend on the measurement rate $\gamma_{k}$. The reason for this is that it has been seen that the CPHD update formula already provides a likelihood for the update of the measurement rate parameters with the multiplicative terms $G_{\mathbf{z}}^{|\mathbf{W}|}(0 \mid \cdot)$ which depend on $\gamma_{k}$. In fact, these terms come directly from the term $P_{\mathbf{z}}\left(N_{\mathrm{T}, k} \mid \xi\right)=P_{\mathbf{z}}\left(N_{\mathrm{T}, k} \mid \gamma\right)$ in the set likelihood (3).

The following two assumptions are standard in many target tracking applications, see e.g., [1].

Assumption 6: Each target's kinematical state follows a linear Gaussian dynamical model.

Assumption 7: The sensor has a linear Gaussian measurement model.

In particular, we use the dynamical model suggested in [4],

$$
\mathbf{x}_{k+1}=\left(F_{k+1 \mid k} \otimes \mathbf{I}_{d}\right) \mathbf{x}_{k}+\mathbf{w}_{k+1}
$$

where $\mathbf{w}_{k+1}$ is zero mean Gaussian process noise with covariance $\Delta_{k+1 \mid k}=\mathbf{Q}_{k+1 \mid k} \otimes X_{k+1}, \mathbf{I}_{d}$ is an identity matrix of dimension $d$, and $F_{k+1 \mid k}$ and $\mathbf{Q}_{k+1 \mid k}$ are [4],

$$
\begin{aligned}
F_{k+1 \mid k} & =\left[\begin{array}{ccc}
1 & T_{s} & \frac{1}{2} T_{s}^{2} \\
0 & 1 & T_{s} \\
0 & 0 & e^{-T_{s} / \theta}
\end{array}\right], \\
\mathbf{Q}_{k+1 \mid k} & =\Sigma^{2}\left(1-e^{-2 T_{s} / \theta}\right) \operatorname{diag}\left(\left[\begin{array}{lll}
0 & 0 & 1
\end{array}\right]\right),
\end{aligned}
$$

where $T_{s}$ is the sampling time, $\Sigma$ is the scalar acceleration standard deviation and $\theta$ is the maneuver correlation time.

The measurement model is also suggested in [4],

$$
\mathbf{z}_{k}=\left(H_{k} \otimes \mathbf{I}_{d}\right) \mathbf{x}_{k}+\mathbf{e}_{k},
$$

where $H_{k}=\left[\begin{array}{lll}1 & 0 & 0\end{array}\right]$ and $\mathbf{e}_{k}$ is white Gaussian noise with covariance given by the target extension matrix $X_{k}$.

\section{THE GGIW-CPHD FILTER}

In this section we present a gamma Gaussian inverse Wishart implementation of the extended target CPHD filter.

\section{A. Assumptions}

In order to derive prediction and correction equations for the GGIW-CPHD filter, a number of assumptions are made here in addition to the assumptions already described.

Assumption 8: The current estimated PHD $D_{k \mid k}(\cdot)$ is an unnormalized mixture of GGIW distributions,

$$
D_{k \mid k}\left(\xi_{k}\right) \approx \sum_{j=1}^{J_{k \mid k}} w_{k \mid k}^{(j)} \mathcal{G} \mathcal{G} \mathcal{I} \mathcal{W}\left(\xi_{k} ; \zeta_{k \mid k}^{(j)}\right),
$$

where $J_{k \mid k}$ is the number of components, $w_{k \mid k}^{(j)}$ is the weight of the $j$ :th component, and $\zeta_{k \mid k}^{(j)}$ is the density parameter of the $j$ :th component.

Assumption 9: The intensity of the birth RFS is an unnormalized mixture of GGIW distributions.

Assumption 10: The survival probability is state independent, i.e. $P_{\mathrm{S}}\left(\xi_{k}\right)=P_{\mathrm{S}}$.

Similarly to [22], [24], an assumption is made concerning the probability of detection $P_{\mathrm{D}}(\cdot)$.

Assumption 11: The following approximation about $P_{\mathrm{D}}(\cdot)$ holds for all $\xi_{k}$

$$
\begin{aligned}
& P_{\mathrm{D}}\left(\xi_{k}\right) \mathcal{G} \mathcal{G} \mathcal{I} \mathcal{W}\left(\xi_{k} ; \zeta_{k \mid k-1}^{(j)}\right) \\
& \approx P_{\mathrm{D}}\left(\zeta_{k \mid k-1}^{(j)}\right) \mathcal{G} \mathcal{G} \mathcal{I} \mathcal{W}\left(\xi_{k} ; \zeta_{k \mid k-1}^{(j)}\right) .
\end{aligned}
$$

In Assumption 11 the approximation (26) is trivially satisfied when $P_{\mathrm{D}}(\cdot)=P_{\mathrm{D}}$, i.e., when $P_{\mathrm{D}}(\cdot)$ is constant. In general, Assumption 11 holds approximately when the function $P_{\mathrm{D}}(\cdot)$ does not vary much in the uncertainty zone of a target in the augmented state space $\xi_{k}$. This is true either when $P_{\mathrm{D}}(\cdot)$ is a sufficiently smooth function, or when the signal to noise ratio (SNR) is high enough such that the uncertainty zone is sufficiently small. 


\section{B. Prediction}

The PHD corresponding to prediction of existing targets is given by

$$
D_{k+1 \mid k}\left(\xi_{k+1}\right)=\int P_{\mathrm{S}}\left(\xi_{k}\right) p\left(\xi_{k+1} \mid \xi_{k}\right) D_{k \mid k}\left(\xi_{k}\right) \mathrm{d} \xi_{k} .
$$

Utilizing (25) and Assumptions 4 and 10, the integral simplifies to

$$
\begin{aligned}
\sum_{j=1}^{J_{k \mid k}} P_{\mathrm{S}} w_{k \mid k}^{(j)} \underbrace{\int \mathcal{G \mathcal { M }}\left(\gamma_{k} ; \alpha_{k \mid k}^{(j)}, \beta_{k \mid k}^{(j)}\right) p^{\gamma}\left(\gamma_{k+1} \mid \gamma_{k}\right) \mathrm{d} \gamma_{k}}_{\text {Measurement rate }} \\
\times \underbrace{\left.\int \begin{array}{r}
\mathcal{N}\left(\mathbf{x}_{k} ; m_{k \mid k}^{(j)}, P_{k \mid k}^{(j)} \otimes X_{k+1}\right) \\
\times p^{\mathbf{x}}\left(\mathbf{x}_{k+1} \mid X_{k+1}, \mathbf{x}_{k}\right)
\end{array}\right) \mathrm{d} \mathbf{x}_{k}}_{\text {Kinematics }} \\
\times \underbrace{\int \mathcal{I} \mathcal{W}_{d}\left(X_{k} ; v_{k \mid k}^{(j)}, V_{k \mid k}^{(j)}\right) p^{X}\left(X_{k+1} \mid X_{k}\right) \mathrm{d} X_{k}}_{\text {Extension }} .
\end{aligned}
$$

Using the linear Gaussian model given in (22), the prediction for the kinematical part becomes [4]

$$
\begin{aligned}
& \int \mathcal{N}\left(\mathbf{x}_{k} ; m_{k \mid k}^{(j)}, P_{k \mid k}^{(j)} \otimes X_{k+1}\right) p^{\mathbf{x}}\left(\mathbf{x}_{k+1} \mid X_{k+1}, \mathbf{x}_{k}\right) \mathrm{d} \mathbf{x}_{k} \\
& =\mathcal{N}\left(\mathbf{x}_{k+1} ; m_{k+1 \mid k}^{(j)}, P_{k+1 \mid k}^{(j)} \otimes X_{k+1}\right)
\end{aligned}
$$

where

$$
\begin{aligned}
m_{k+1 \mid k}^{(j)} & =\left(F_{k+1 \mid k} \otimes \mathbf{I}_{d}\right) m_{k \mid k}^{(j)} \\
P_{k+1 \mid k}^{(j)} & =F_{k+1 \mid k} P_{k \mid k}^{(j)} F_{k+1 \mid k}^{\mathrm{T}}+\mathbf{Q}_{k+1 \mid k} .
\end{aligned}
$$

The integrals that correspond to the measurement rate and extension are less straightforward to solve. For measurement rate prediction, as in [37] we use exponential forgetting with forgetting factor $\frac{1}{\eta_{k}}$,

$$
\alpha_{k+1 \mid k}^{(j)}=\frac{\alpha_{k \mid k}^{(j)}}{\eta_{k}}, \quad \beta_{k+1 \mid k}^{(j)}=\frac{\beta_{k \mid k}^{(j)}}{\eta_{k}},
$$

where $\eta_{k}>1$. This prediction has an effective window of length $w_{e}=\frac{1}{1-1 / \eta_{k}}=\frac{\eta_{k}}{\eta_{k}-1}$.

For the extension, we apply the same heuristic approach as in [4], i.e., the predicted degrees of freedom and inverse scale matrix are approximated by

$$
\begin{aligned}
v_{k+1 \mid k}^{(j)} & =e^{-T_{s} / \tau} v_{k \mid k}^{(j)}, \\
V_{k+1 \mid k}^{(j)} & =\frac{v_{k+1 \mid k}^{(j)}-d-1}{v_{k \mid k}^{(j)}-d-1} V_{k \mid k}^{(j)},
\end{aligned}
$$

where $T_{s}$ is the sample time and $\tau$ is a temporal decay constant. Thus, the PHD (27) corresponding to predicted existing targets is

$$
D_{k+1 \mid k}\left(\xi_{k+1}\right)=\sum_{j=1}^{J_{k \mid k}} w_{k+1 \mid k}^{(j)} \mathcal{G} \mathcal{G} \mathcal{I} \mathcal{W}\left(\xi_{k+1} ; \zeta_{k+1 \mid k}^{(j)}\right),
$$

where $w_{k+1 \mid k}^{(j)}=P_{\mathrm{S}} w_{k \mid k}^{(j)}$, and the predicted parameters $\zeta_{k+1 \mid k}^{(j)}$ are given by (30), (31), and (32).
The birth PHD

$$
D_{k}^{b}\left(\xi_{k}\right)=\sum_{j=1}^{J_{b, k}} w_{b, k}^{(j)} \mathcal{G} \mathcal{G} \mathcal{I} \mathcal{W}\left(\xi_{k} ; \zeta_{b, k}^{(j)}\right),
$$

represents new targets that appear at time step $k$. For the sake of simplicity, as in [24] target spawning is omitted. The full predicted PHD $D_{k+1 \mid k}\left(\xi_{k+1}\right)$ is the sum of the PHD of predicted existing targets (33) and the birth PHD (34), and contains a total of $J_{k+1 \mid k}=J_{k \mid k}+J_{b, k+1}$ GGIW components.

The cardinality distribution is predicted as in [31],

$$
\begin{aligned}
P_{k+1 \mid k}(n)= & \sum_{j=0}^{n} P_{b, k}(n-j) \\
& \times \sum_{\ell=j}^{\infty} \frac{\ell !}{j !(\ell-j) !} P_{k \mid k}(\ell) P_{\mathrm{S}, k}^{j}\left(1-P_{\mathrm{S}, k}\right)^{\ell-j},
\end{aligned}
$$

where $P_{b, k}(n)$ is the cardinality distribution of birth.

\section{Correction}

The correction has the following steps:

1) For all components $j$, and all sets $\mathbf{W}$ in all partitions $\mathcal{P}$ of $\mathbf{Z}_{k}$ : First compute the centroid measurement, scatter matrix, innovation factor, gain vector, innovation vector and innovation matrix,

$$
\begin{aligned}
\overline{\mathbf{z}}_{k}^{\mathbf{W}} & =\frac{1}{|\mathbf{W}|} \sum_{\mathbf{z}_{k}^{(i)} \in \mathbf{W}} \mathbf{z}_{k}^{(i)} \\
Z_{k}^{\mathbf{W}} & =\sum_{\mathbf{z}_{k}^{(i)} \in \mathbf{W}}\left(\mathbf{z}_{k}^{(i)}-\overline{\mathbf{z}}_{k}^{\mathbf{W}}\right)\left(\mathbf{z}_{k}^{(i)}-\overline{\mathbf{z}}_{k}^{\mathbf{W}}\right)^{\mathrm{T}} \\
S_{k \mid k-1}^{(j), \mathbf{W}} & =H_{k} P_{k \mid k-1}^{(j)} H_{k}^{\mathrm{T}}+\frac{1}{|\mathbf{W}|} \\
K_{k \mid k-1}^{(j), \mathbf{W}} & =P_{k \mid k-1}^{(j)} H_{k}^{\mathrm{T}}\left(S_{k \mid k-1}^{(j), \mathbf{W}}\right)^{-1} \\
\varepsilon_{k \mid k-1}^{(j), \mathbf{W}} & =\overline{\mathbf{z}}_{k}^{\mathbf{W}}-\left(H_{k} \otimes \mathbf{I}_{d}\right) m_{k \mid k-1}^{(j)} \\
N_{k \mid k-1}^{(j), \mathbf{W}} & =\left(S_{k \mid k-1}^{(j), \mathbf{W}}\right)^{-1} \varepsilon_{k \mid k-1}^{(j), \mathbf{W}}\left(\varepsilon_{k \mid k-1}^{(j), \mathbf{W}}\right)^{\mathrm{T}}
\end{aligned}
$$

and then compute the posterior GGIW parameters

$$
\begin{aligned}
\alpha_{k \mid k}^{(j), \mathbf{W}} & =\alpha_{k \mid k-1}^{(j)}+|\mathbf{W}| \\
\beta_{k \mid k}^{(j), \mathbf{W}} & =\beta_{k \mid k-1}^{(j)}+1 \\
m_{k \mid k}^{(j), \mathbf{W}} & =m_{k \mid k-1}^{(j)}+\left(K_{k \mid k-1}^{(j), \mathbf{W}} \otimes \mathbf{I}_{d}\right) \varepsilon_{k \mid k-1}^{(j), \mathbf{W}} \\
P_{k \mid k}^{(j), \mathbf{W}} & =P_{k \mid k-1}^{(j)}-K_{k \mid k-1}^{(j), \mathbf{W}} S_{k \mid k-1}^{(j), \mathbf{W}}\left(K_{k \mid k-1}^{(j), \mathbf{W}}\right)^{\mathrm{T}} \\
v_{k \mid k}^{(j), \mathbf{W}} & =v_{k \mid k-1}^{(j)}+|\mathbf{W}| \\
V_{k \mid k}^{(j), \mathbf{W}} & =V_{k \mid k-1}^{(j)}+N_{k \mid k-1}^{(j), \mathbf{W}}+Z_{k}^{\mathbf{W}}
\end{aligned}
$$

2) Calculate $\rho$, according to (12a), as

$$
\rho=\sum_{j=1}^{J_{k \mid k-1}} \bar{w}_{k \mid k-1}^{(j)}\left(1-P_{\mathrm{D}}^{(j)}+P_{\mathrm{D}}^{(j)} G_{\mathbf{z}}(0, j)\right)
$$


where

$$
G_{\mathbf{z}}(0, j)=\left(\frac{\beta_{k \mid k-1}^{(j)}}{\beta_{k \mid k-1}^{(j)}+1}\right)^{\alpha_{k \mid k-1}^{(j)}}
$$

is the expected probability that the $j$ :th component did not cause any detections, and

$$
\bar{w}_{k \mid k-1}^{(j)} \triangleq \frac{w_{k \mid k-1}^{(j)}}{\sum_{\ell=1}^{J_{k \mid k-1}} w_{k \mid k-1}^{(\ell)}}
$$

for $j=1, \ldots, J_{k \mid k-1}$ are the normalized prior PHD weights.

3) Calculate $\eta_{\mathbf{W}}$ for all sets $\mathbf{W}$ in all partitions $\mathcal{P}$ of $\mathbf{Z}_{k}$ according to (12b), as follows.

$$
\eta_{\mathbf{W}}=\sum_{j=1}^{J_{k \mid k-1}} \bar{w}_{k \mid k-1}^{(j)} P_{\mathrm{D}}^{(j)} \frac{\mathcal{L}_{k}^{(j), \mathbf{W}}}{\mathcal{L}_{\mathrm{FA}}^{\mathbf{W}}}
$$

where

$$
\begin{aligned}
& \mathcal{L}_{k}^{(j), \gamma}=\frac{\Gamma\left(\alpha_{k \mid k}^{(j),|\mathbf{W}|}\right)\left(\beta_{k \mid k-1}^{(j)}\right)^{\alpha_{k \mid k-1}^{(j)}}}{\Gamma\left(\alpha_{k \mid k-1}^{(j)}\right)\left(\beta_{k \mid k}^{(j), \mathbf{W}}\right)^{\alpha_{k \mid k}^{(j),|\mathbf{W}|}}} \\
& \mathcal{L}_{k}^{(j), \mathbf{x}, X}=\frac{\left(\pi^{|\mathbf{W}|}|\mathbf{W}|\right)^{-\frac{d}{2}}}{\left(S_{k \mid k-1}^{(j), \mathbf{W}}\right)^{\frac{d}{2}}} \frac{\left|V_{k \mid k-1}^{(j)}\right|^{\frac{v_{k \mid k-1}^{(j)}}{2}}}{\left|V_{k \mid k}^{(j, \mathbf{W})}\right|^{\frac{v_{k \mid k}^{(j), \mathbf{W}}}{2}}} \frac{\Gamma_{d}\left(\frac{v_{k \mid k}^{(j), \mathbf{W}}}{2}\right)}{\Gamma_{d}\left(\frac{v_{k \mid k-1}^{(j)}}{2}\right)} \\
& \mathcal{L}_{k}^{(j), \mathbf{W}}=\mathcal{L}_{k}^{(j), \gamma} \mathcal{L}_{k}^{(j), \mathbf{x}, X} \\
& \mathcal{L}_{\mathrm{FA}}^{\mathrm{W}}=\prod_{\mathbf{z} \in \mathbf{W}} p_{\mathrm{FA}}(\mathbf{z})
\end{aligned}
$$

In the GIW likelihood (38c), $|V|$ denotes the determinant of the matrix $V$, and $|\mathbf{W}|$ is the number of measurements in the cell $\mathbf{W}$. The derivation of the GIW likelihood (38c) is given in [24]. The GGIW likelihood (38d) is the product of the measurement rate likelihood (38b) and the GIW likelihood (38c).

4) Calculate the coefficients $\iota_{\mathcal{P}, \mathbf{W}}, \chi_{\mathcal{P}, \mathbf{W}}, \psi_{\mathcal{P}, \mathbf{W}}, \kappa$ and $\sigma_{\mathcal{P}, \mathbf{W}}$ for all sets $\mathbf{W}$ and all partitions $\mathcal{P}$ using the formulas (12c), (12d), (12e), (12f) and (12g) respectively.

5) Calculate the posterior weights $w_{k \mid k}^{(j), \mathcal{P}, \mathbf{W}}$ as

$$
w_{k \mid k}^{(j), \mathcal{P}, \mathbf{W}}=\frac{\bar{w}_{k \mid k-1}^{(j)} P_{\mathrm{D}}^{(j)} \sigma_{\mathcal{P}, \mathbf{W}} \frac{\mathcal{L}_{k}^{(j), \mathbf{w}}}{\mathcal{L}_{\mathrm{FA}}^{\mathrm{W}}}}{\sum_{\mathcal{P} \angle \mathbf{Z}} \sum_{\mathbf{W} \in \mathcal{P}} \psi_{\mathcal{P}, \mathbf{W}} \iota \mathcal{P}, \mathbf{W}}
$$

The posterior PHD is a GGIW mixture

$$
\begin{aligned}
D_{k \mid k}\left(\xi_{k}\right)= & \sum_{\mathcal{P} \angle \mathbf{Z}_{k}} \sum_{\mathbf{W} \in \mathcal{P}} w_{k \mid k}^{(j), \mathcal{P}, \mathbf{W}} \mathcal{G} \mathcal{G} \mathcal{I} \mathcal{W}\left(\xi_{k} ; \zeta_{k \mid k}^{(j), \mathbf{W}}\right) \\
& +D_{k \mid k}^{\mathrm{ND}}\left(\xi_{k}\right)
\end{aligned}
$$

where $D_{k \mid k}^{\mathrm{ND}}(\cdot)$ is given by

$$
\begin{aligned}
D_{k \mid k}^{\mathrm{ND}}\left(\xi_{k}\right)= & \kappa \sum_{j=1}^{J_{k \mid k-1}} \bar{w}_{k \mid k-1}^{(j)}\left(1-P_{\mathrm{D}}^{(j)}\right) \mathcal{G} \mathcal{G} \mathcal{I} \mathcal{W}\left(\xi_{k} ; \zeta_{k \mid k-1}^{(j)}\right) \\
& +\kappa \sum_{j=1}^{J_{k \mid k-1}} \bar{w}_{k \mid k-1}^{(j)} P_{\mathrm{D}}^{(j)}\left(\frac{\beta_{k \mid k-1}^{(j)}}{\beta_{k \mid k-1}^{(j)}+1}\right)^{\alpha_{k \mid k-1}^{(j)}} \\
& \times \mathcal{G} \mathcal{G} \mathcal{I} \mathcal{W}\left(\xi_{k} ; \bar{\zeta}_{k \mid k-1}^{(j)}\right) .
\end{aligned}
$$

The first summation on the right hand side of (41) represents the cases where a target is not detected and the second summation deal with the cases when the target is detected but it does not generate any measurements. All of the modified parameters $\bar{\zeta}_{k \mid k-1}^{(j)}$ in the second summation are the same as the parameters $\zeta_{k \mid k-1}^{(j)}$ except that $\bar{\beta}_{k \mid k-1}^{(j)}=\beta_{k \mid k-1}^{(j)}+1$.

The calculation of the updated cardinality distribution $P_{k \mid k}(\cdot)$ is straightforward with (11) using the quantities calculated above.

\section{Measurement Set Partitioning}

Note that the presented ET-CPHD filter, like the ET-PHD [21], requires all partitions of the current measurement set for its update. A partition is a division of the measurement set into non-empty subsets called cells. Each cell can be interpreted as containing measurements that all stem from the same source, either a target or a clutter source.

As the total number of measurements grows, the number of possible partitons grows very large. Even with as little as five measurements there are 120 possible partitions, hence considering all partitions is computationally infeasible and approximations are necessary. In [22], [23] it was shown that the set of all partitions can efficiently be approximated with a subset of partitions, provided that the subset contains the most likely partitions. The partitioning methods in [22], [23] puts upper bound constraints on the distances between measurements that are put into the same cells $\mathbf{W}$ in a partition $\mathcal{P}$. These partitioning methods have been shown to reduce the number of partitions that have to be considered by several orders of magnitude, while sacrificing as little tracking performance as possible [10], [22]-[24], [34], [37].

A careful investigation of the presented ET-CPHD filter update equations makes it evident that for correct operation, the ET-CPHD filter requires partitions that would include the false alarms in a single cell. As an illustration, suppose that the current measurement set is composed of two clusters of closely spaced target generated measurements, and 10 individual well separated false alarm measurement, see Fig. 1a. In this case, the partitioning algorithms used would most probably supply a single partition of 12 cells: two cells containing the respective target originated measurements, and the other ten cells containing the false alarms, see Fig. 1b. In the update of the cardinality distribution (11), the probabilities for all $n$ smaller than $|\mathcal{P}|-1$ are set to zero. Hence the result would be a grossly incorrect cardinality estimate, which would lead to a lot of false tracks. 


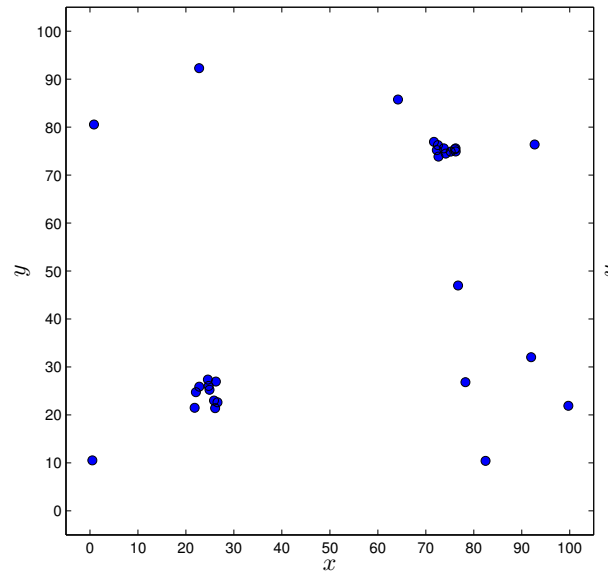

(a)

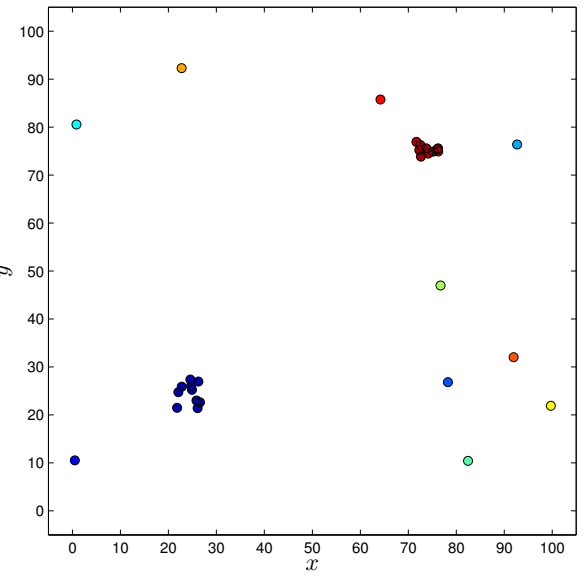

(b)

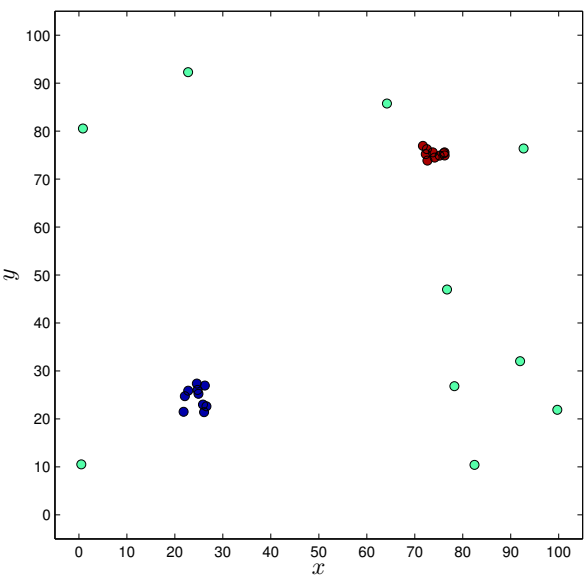

(c)

Fig. 1. Partitioning illustration. (a) Two targets at $\left[\begin{array}{ll}25 & 25\end{array}\right]^{\mathrm{T}}$ and $\left[\begin{array}{ll}75 & 75\end{array}\right]^{\mathrm{T}}$ with 10 measurements each, and 10 uniformly distributed clutter measurements (b) Measurement set partition obtained using the method from [22], [24]. Different cells are illustrated using different colors. The true target measurements have correctly been allocated to two cells, and the clutter measurements belong to individual cells. (c) Cells with less than or equal to $N_{l o w}=1$ measurement have been merged to a single cell.

This problem is a direct manifestation of the approximation of the set of all partitions with a limited number of distance based partitions. Note that if the full set of partitions was used, there would always be a partition that puts the target generated measurements into individual cells, and all clutter measurements into a single cell.

The problem can be solved in at least two alternative ways. The first solution is to derive the ET-CPHD filter in a slightly different way, which results in two levels of partitioning in the update. This is done in [39] where the final formulae for the ET-CPHD filter update, though equivalent to the update presented in this manuscript, requires partitioning of all cells in all partitions of the measurement set. Distance based partitioning in both levels then solves the problem. On the other hand, this type of solution causes other implementational difficulties. First, the computational overload increases unnecessarily with two levels of partitioning which is rather inefficient. Secondly, and more importantly, such an implementation results in some PHD components with negative weights $w_{k \mid k}^{(j)}$. The resulting PHD remains valid thanks to the identical PHD components whose weights always sum up to their true positive values.

The second solution, which is used in the paper, is a variant of the distance partitioning method presented in [22], [24]. Due to space considerations, only the necessary modifications are discussed here and the reader is referred to [22], [24] for the basic algorithm. The distance partitioning method would, without changes, put the false alarms into individual cells, as illustrated in Fig.1b. The reason is that they are in general isolated measurements far away from the target generated measurement clusters. In the present work the method has therefore been modified in the sense that an additional partition is created, for partitions computed by the distance partitioning method, that merge all the cells that contain $|\mathbf{W}| \leq N_{\text {low }}$ measurements into a single cell. This is illustrated for $N_{\text {low }}=1$ in Fig.1c. In this way, there remains still a single level of partitioning, which is in fact the same computational overload as the ET-PHD filter, but the set of distance based partitions are further processed to compose new partitions that would solve the problem of cardinality overestimation in the case of high number of false alarms.

\section{E. Pruning and Merging}

The usual techniques of merging and pruning, see [16] for details on GM-PHD, must be applied to reduce the exponential growth of the number of GGIW components. Merging methods specific to GGIW mixtures can be found in [37], [38]. For space consideration these algorithms are not repeated here.

\section{F. Target extraction and maintaining target tracks}

Similarly to [16], [22], [24] targets are extracted from the PHD intensity by taking the components $i$ for which it holds $w_{k \mid k}^{(i)}>0.5$. Let the set of extracted targets be

$$
\begin{gathered}
\hat{\mathbf{X}}_{k \mid k}=\left\{\hat{\xi}_{k \mid k}^{(i)}\right\}_{i=1}^{\hat{N}_{x, k}}, \hat{\xi}_{k \mid k}^{(i)}=\left(\hat{\gamma}_{k \mid k}^{(i)}, \hat{\mathbf{x}}_{k \mid k}^{(i)}, \hat{X}_{k \mid k}^{(i)}\right), \\
\hat{\gamma}_{k \mid k}^{(i)}=\mathrm{E}\left[\gamma_{k}\right], \hat{\mathbf{x}}_{k \mid k}^{(i)}=\mathrm{E}\left[\mathbf{x}_{k}\right], \hat{X}_{k \mid k}^{(i)}=\mathrm{E}\left[X_{k}\right],
\end{gathered}
$$

where the expected values are taken with respect to the $i$ :th GGIW distribution.

Note that this GGIW implementation of the ET-CPHD filter does not maintain target identities over time. For the point target GM-PHD filter [16], methods to maintain target identities are presented in e.g., [40], [41]. The presented GGIW CPHD filter can easily be augmented in a similar manner to provide target identities over time. In this case, the estimates of the measurement rate and the extension would aid in the process of maintaining target identities over time.

\section{Vi. Performance Evaluation}

In this section, we discuss the metrics used in order to evaluate the performance of the GGIW-CPHD filter. It is of 
interest to compare the set of extracted targets (42) to the true set of targets,

$$
\mathbf{X}_{k}=\left\{\xi_{k}^{(j)}\right\}_{j=1}^{N_{\xi, k}}, \quad \xi_{k}^{(j)}=\left(\gamma_{k}^{(j)}, \mathbf{x}_{k}^{(j)}, X_{k}^{(j)}\right) .
$$

Here we use a variant of the optimal sub-pattern assignment (OSPA) metric [42]. The distance between $\xi_{k}^{(j)}$ and $\hat{\xi}_{k \mid k}^{(i)}$ is decomposed as

$$
d\left(\xi_{k}^{(j)}, \hat{\xi}_{k \mid k}^{(i)}\right)=\frac{w_{\gamma}}{c_{\gamma}} d_{j, i}^{\left(c_{\gamma}\right)}+\frac{w_{\mathbf{x}}}{c_{\mathbf{x}}} d_{j, i}^{\left(c_{\mathbf{x}}\right)}+\frac{w_{X}}{c_{X}} d_{j, i}^{\left(c_{X}\right)},
$$

where $w_{\gamma}+w_{\mathbf{x}}+w_{X}=1$, and

$$
\begin{aligned}
& d_{j, i}^{\left(c_{\gamma}\right)}=\min \left(c_{\gamma},\left|\gamma_{k}^{(j)}-\hat{\gamma}_{k \mid k}^{(i)}\right|\right), \\
& d_{j, i}^{\left(c_{\mathbf{x}}\right)}=\min \left(c_{\mathbf{x}},\left\|\mathbf{x}_{k}^{(j)}-\hat{\mathbf{x}}_{k \mid k}^{(i)}\right\|_{2}\right), \\
& d_{j, i}^{\left(c_{X}\right)}=\min \left(c_{X},\left\|X_{k}^{(j)}-\hat{X}_{k \mid k}^{(i)}\right\|_{F}\right),
\end{aligned}
$$

where $|\cdot|$ is the absolute value, $\|\cdot\|_{2}$ is the Euclidean norm, and $\|\cdot\|_{F}$ is the Frobenius norm. The constants $c_{\gamma}, c_{\mathbf{x}}$ and $c_{X}$ are chosen such that they correspond to the maximum expected error for the measurement rate, kinematical state, and extension state, respectively.

An optimal assignment $\bar{\pi}$ of order $p$ with cut-off $c$ is given by

$$
\begin{gathered}
\bar{\pi}=\underset{\pi \in \Pi_{n}}{\arg \min } \sum_{i=1}^{N_{\xi, k}}\left(d_{j, i}^{(c)}\right)^{p}, \\
d_{j, i}^{(c)}=\min \left(c, d\left(\xi_{k}^{(j)}, \hat{\xi}_{k \mid k}^{(i)}\right)\right) .
\end{gathered}
$$

The multiple extended target tracking performance is presented in terms of the following quantity,

$$
\bar{d}_{p}^{(c)}=\left(\frac{1}{n} \sum_{i=1}^{N_{\xi, k}}\left(d_{i, \bar{\pi}(i)}^{(c)}\right)^{p}+c^{p}\left(\hat{N}_{\xi, k}-N_{\xi, k}\right)\right)^{\frac{1}{p}} .
$$

A cardinality estimate can be obtained in a few different ways. One estimate is the number of extracted targets, $\hat{N}_{\xi, k}$, another is the sum of weights $\Sigma_{i} w_{k \mid k}^{(i)}$. For the CPHD filter, an estimate can also be taken as the maximum likelihood estimate from the cardinality distribution,

$$
N_{\xi, k}^{C}=\underset{n}{\arg \max } P_{k \mid k}(n) .
$$

In the sections below where our results are presented, we will compare the true cardinality with $\Sigma_{i} w_{k \mid k}^{(i)}$ for the PHD filter, and $N_{\xi, k}^{C}$ for the CPHD filter.

\section{Simulation Results}

In this section we present results from a simulation study that compares the GGIW-CPHD filter to a GGIW implementation of the ET-PHD filter [21], called GGIW-PHD filter. The GGIWPHD filter is a variant of the GIW-PHD filter presented in [24], where measurement rate estimation has been added as outlined in [37].

Implementing the GGIW-CPHD filter can be cumbersome, to facilitate reproducibility and future comparisons a MATLAB implementation of the GGIW-CPHD filter is freely available for research purposes. It can be acquired by contacting the authors.

\section{A. Target Tracking Setup}

Two different scenarios were simulated, one with two targets and one with four targets. The true extensions are

$$
X_{k}^{(i)}=R_{k}^{(i)} \operatorname{diag}\left(\left[A_{i}^{2} a_{i}^{2}\right]\right)\left(R_{k}^{(i)}\right)^{\mathrm{T}},
$$

where $R_{k}^{(i)}$ is rotation matrix applied such that the $i$ :th extension's major axis is aligned with the $i$ :th target's direction of motion at time step $k$, and $A_{i}$ and $a_{i}$ are the length of the major and minor axes, respectively. True target measurements were generated with Poisson rates $\gamma_{k}^{(i)}$.

In the scenario with four targets, the major and minor axes, and measurement rates, are

$$
\begin{aligned}
& A_{1}=5, \quad a_{1}=3, \quad \gamma_{k}^{(1)}=10, \\
& A_{2}=5 \sqrt{2}, \quad a_{2}=3 \sqrt{2}, \quad \gamma_{k}^{(2)}=20 \text {, } \\
& A_{3}=5 \sqrt{1.5}, \quad a_{3}=3 \sqrt{1.5}, \quad \gamma_{k}^{(3)}=15 \text {, } \\
& A_{4}=5 \sqrt{2.5}, \quad a_{4}=3 \sqrt{2.5}, \quad \gamma_{k}^{(4)}=25 \text {. }
\end{aligned}
$$

The scenario is 200 time steps, the targets appear at time $t_{b}^{(i)}$ and disappear at time $t_{d}^{(i)}$,

$$
\begin{aligned}
& t_{b}^{(1)}=10, \quad t_{b}^{(2)}=35, \quad t_{b}^{(3)}=60, \quad t_{b}^{(4)}=85, \\
& t_{d}^{(1)}=110, \quad t_{d}^{(2)}=135, \quad t_{d}^{(3)}=160, \quad t_{d}^{(4)}=185 .
\end{aligned}
$$

The true tracks of the kinematical states are shown in Fig. 2a, the surveilance area is $[-200,200] \times[-200,200]$.

In the scenario with two targets, the major and minor axes, and measurement rates, are

$$
\begin{array}{lll}
A_{1}=20, & a_{1}=5, & \gamma_{k}^{(1)}=20, \\
A_{2}=10, & a_{2}=2.5, & \gamma_{k}^{(2)}=10 .
\end{array}
$$

Both targets are present during the entire simulation (100 time steps). The true tracks of the kinematical states are shown in Fig. $2 b$, the surveilance area is $\left[-10^{4}, 10^{4}\right] \times\left[-10^{4}, 10^{4}\right]$. This scenario was also used in [24].

In the filter, the motion model parameters are set to $T_{s}=1 \mathrm{~s}$, $\theta=1 \mathrm{~s}, \Sigma=0.1 \mathrm{~m} / \mathrm{s}^{2}$ and $\tau=5 \mathrm{~s}$. For the performance evaluation the parameters were set to $p=1, c=c_{\gamma}+c_{\mathbf{x}}+c_{X}$, $c_{\gamma}=10, c_{\mathbf{x}}=50, c_{X}=50, w_{\gamma}=0.1, w_{\mathbf{x}}=0.8$, and $w_{X}=$ 0.1 . In the partitioning we use $N_{\text {low }} \in\{1,2,3\}$. The birth PHD is set such that the Gaussian mean vectors correspond to the targets' actual starting points. The birth inverse Wishart mean matrices are set to diag ([30 30]) in the scenario with four targets, and $\operatorname{diag}\left(\left[\begin{array}{ll}1 & 1\end{array}\right]\right)$ in the scenario with two targets. The birth measurement rate means are set to 3.5 in the scenario with four targets, and to 15 in the scenario with two targets. Examples of how the birth PHD can be set in an experimental scenario can be found in e.g., [22], [24]. The birth cardinality is set to

$$
P_{b, k}(n)= \begin{cases}0.90 & n=0 \\ 0.05 & n=1 \\ 0.025 & n=2,3 \\ 0 & \text { otherwise }\end{cases}
$$




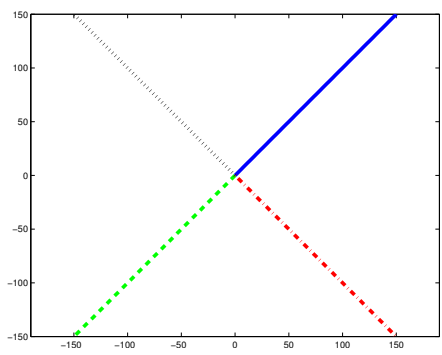

(a)

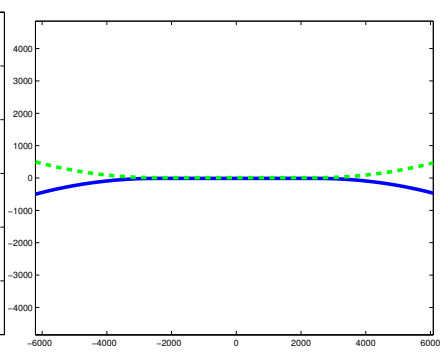

(b)
Fig. 2. True target tracks used in simulations. (a) Four targets that appear and disappear at different times. (b) Two targets that move in parallel.

\section{B. Four Targets}

The scenario was simulated for different probability of detection, 0.80 or 0.99 , and different number of false alarms, $5 / 400^{2}$ or $30 / 400^{2}$ clutter measurements per surveillance volume. The results are shown in Fig. $3 \mathrm{a}-3 \mathrm{~d}$. In comparing the filters we can observe the following:

- In terms of estimating the cardinality, the CPHD filter handles high clutter rate and low probability of detection much better than the PHD filter. This is especially clear when the clutter rate is high and the probability of detection is low at the same time, see Figure $3 \mathrm{~d}$.

- In terms of estimating the extended target state $\xi_{k}$, there is no significant difference between the two filters when probability of detection is high and the clutter rate is low, which can be seen in the OSPA plot in Figure 3a. For the other three parameter settings, the OSPA differences can be attributed to the difference in cardinality estimate.

Both these findings are expected and intuitive - the CPHD filter was introduced because the PHD filter's cardinality estimate has high variance.

\section{Two Targets}

The scenario was simulated with probability of detection 0.99 , and $10 /\left(4 \times 10^{8}\right)$ clutter measurements per surveillance volume. The performance metrics and the cardinality estimates are shown in Fig. 3e. During the parallel motion the PHD filter's performance deteriorates slowly, because a missed detection for one target typically results in target loss, followed by underestimation of cardinality, see [24]. The CPHD filter on the other hand does not have this problem, and as a result has no cardinality error on average, and smaller OSPA errors on average.

Example CPHD filter results from a single simulation run are shown in Figure 4. The motion model does not include information about the direction of motion being aligned with the major axis of the extension, however there is very little difference between the estimated direction of motion and the extimated orientation of the major axis.

\section{Cycle times}

The CPHD filter is, compared to the PHD filter, computationally more complex. In Table I the mean cycle times are given for the scenario with four targets. One cycle consists of

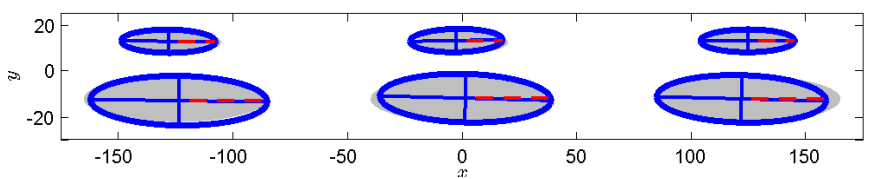

Fig. 4. Example CPHD results showing three time steps from a single simulation run of the scenario with two targets. The true extended targets are shown in gray. The estimates are shown as blue ellipses, with the major and minor axis plotted for increased clarity. The direction of motion is shown with a red dashed line.

measurement set partitioning, prediction, correction, pruning and merging, and target extraction. The results were obtained using a computational server with an Intel Xeon Processor X5675 (61.8GB total memory, 3.06GHz CPU). As expected, the CPHD filter has higher average cycle time.

\section{CONCLUSIONS}

A CPHD filter is given for tracking multiple extended targets, in the presence of clutter and missed detections. A gamma Gaussian inverse Wishart mixture implementation for the derived filter was proposed, and the steps of the algorithm were presented. A simulation study was performed to compare the CPHD filter to the PHD filter. When the targets are spatially separated, the CPHD filter has much better cardinality estimate than the PHD filter, especially in cases with high clutter rate and/or low probability of detection. When the targets are spatially close, the CPHD filter again has better cardinality estimate than the PHD filter, even when the probability of detection is high. In conclusion, the cardinality estimate of the presented CPHD filter is more robust than its PHD counterpart.

An experimental study was not shown in this paper, however some experiments have been performed and the early results confirm the observation that the CPHD filter has a more robust cardinality estimate, see [34] for a detailed comparison. Because there is no ground truth data available, and because the experiment results did not add any significant insight into the CPHD filter other than what was already available from the simulation results, the experiments were omitted in this work. It is the authors' ambition to present a more comprehensive experimental comparison between different multiple extended target trackers in the future.

\section{ACKNOWLEDGMENTS}

The authors gratefully acknowledge the financial support from the Swedish Research Council under the Linnaeus Center (CADICS) and under the frame project grant Extended Target Tracking (ETT) (621-2010-4301), and the financial support from the Swedish Foundation for Strategic Research under the project Collaborative Unmanned Aircraft Systems (CUAS).

TABLE I

CyCle times [s] For the SCENARIO IN Figure 2A. MEAN \pm ONE STANDARD DEVIATION.

\begin{tabular}{|l|cccc|}
\hline$p_{\mathrm{D}}, N_{F A}$ & $0.99,5$ & $0.99,30$ & $0.80,5$ & $0.80,30$ \\
\hline CPHD & $0.20 \pm 0.11$ & $2.65 \pm 0.71$ & $0.29 \pm 0.15$ & $2.20 \pm 0.47$ \\
PHD & $0.08 \pm 0.05$ & $0.59 \pm 0.22$ & $0.17 \pm 0.12$ & $0.73 \pm 0.31$ \\
\hline
\end{tabular}



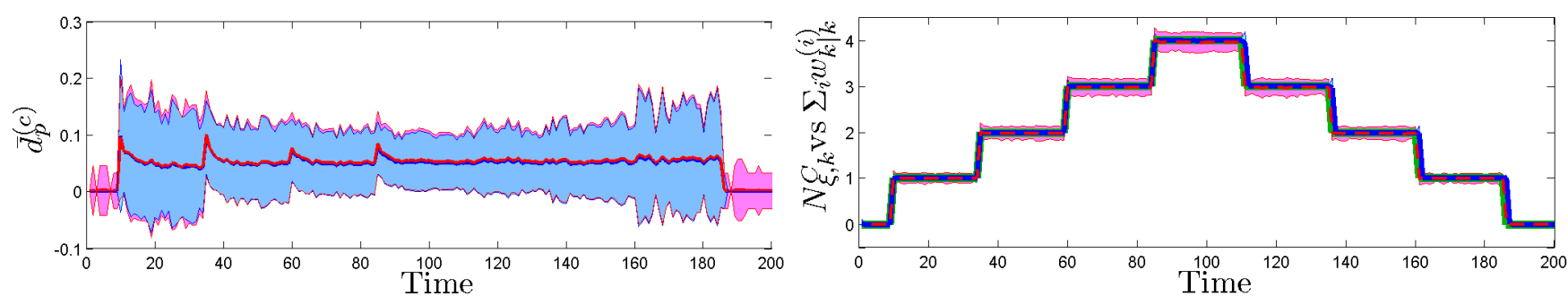

(a) Scenario in Fig. 2a with $p_{\mathrm{D}}=0.99$ and $N_{F A}=5$
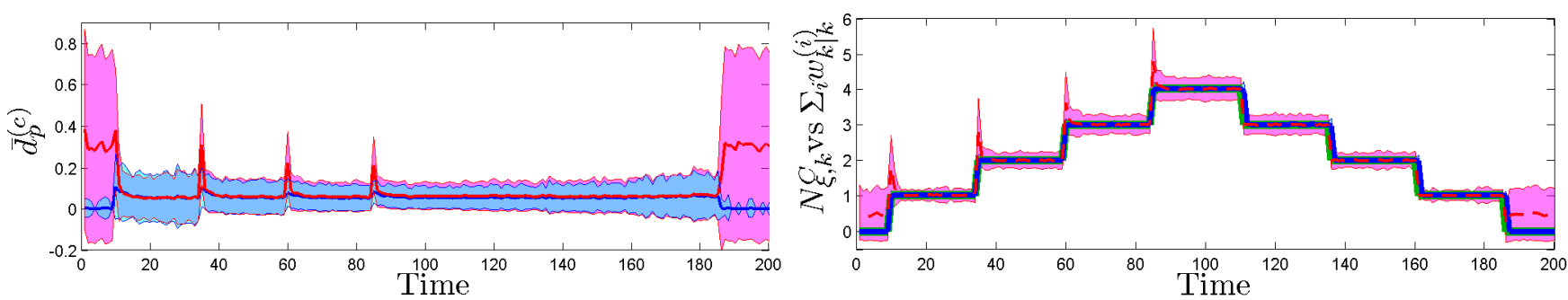

(b) Scenario in Fig. 2a with $p_{\mathrm{D}}=0.99$ and $N_{F A}=30$
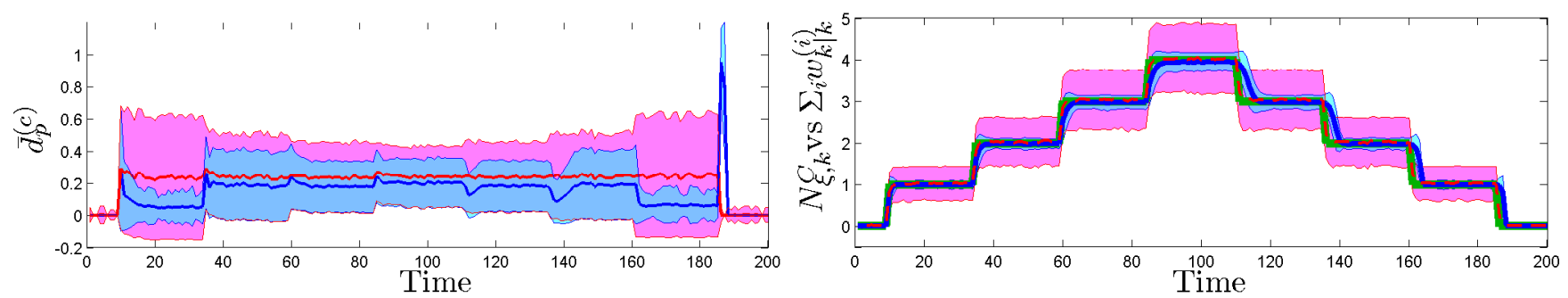

(c) Scenario in Fig. 2a with $p_{\mathrm{D}}=0.80$ and $N_{F A}=5$
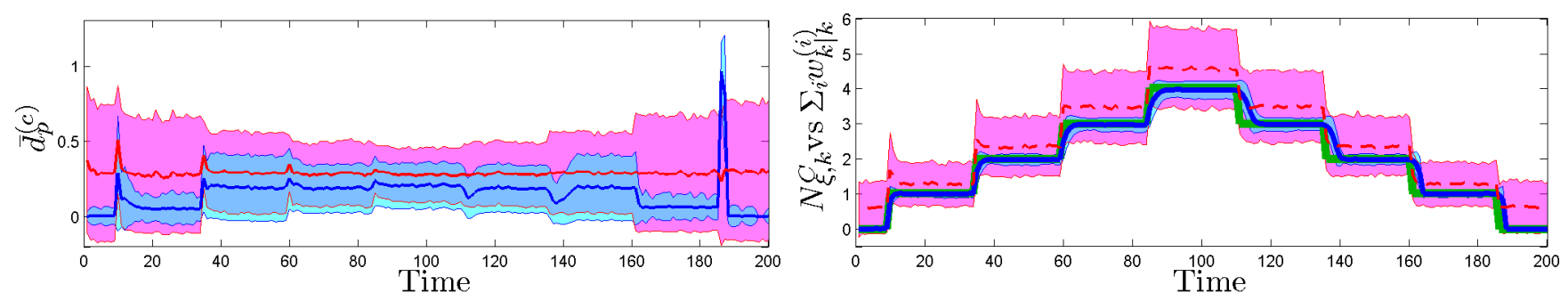

(d) Scenario in Fig. 2a with $p_{\mathrm{D}}=0.80$ and $N_{F A}=30$
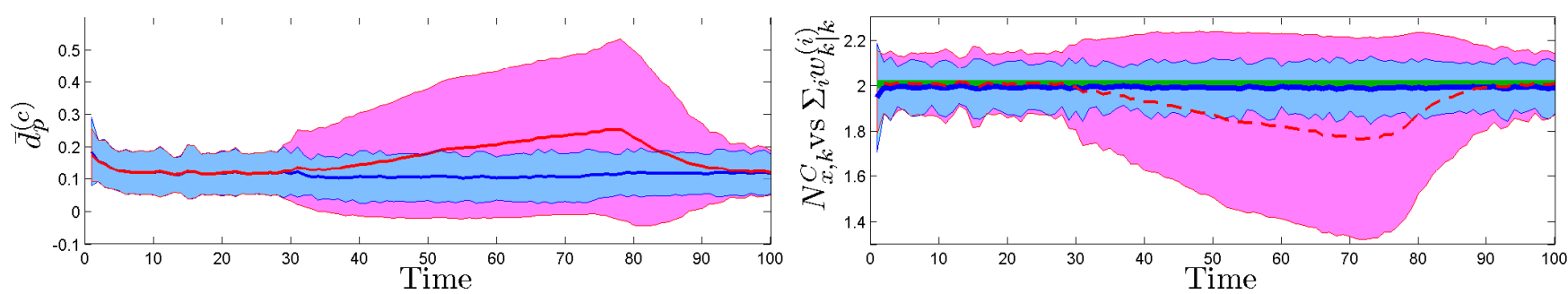

(e) Scenario with two targets in Fig. 2b

Fig. 3. Simulation results. The thick lines show the mean value over $10^{3}$ Monte Carlo simulations, blue is CPHD, red is PHD. The light blue and pink colored areas are mean \pm one standard deviation. Left: OSPA-metric, cf. (47). Right: Comparison of $N_{x, k}^{C}$ for the CPHD filter with the sum of weights for the PHD filter, true cardinality in green.

\section{REFERENCES}

[1] Y. Bar-Shalom and T. E. Fortmann, Tracking and data association, ser. Mathematics in Science and Engineering. San Diego, CA, USA:
Academic Press Professional, Inc., 1987, vol. 179.

[2] K. Gilholm and D. Salmond, "Spatial distribution model for tracking extended objects," IEE Proceedings Radar, Sonar and Navigation, vol. 
152, no. 5, pp. 364-371, Oct. 2005.

[3] K. Gilholm, S. Godsill, S. Maskell, and D. Salmond, "Poisson models for extended target and group tracking," in Proceedings of Signal and Data Processing of Small Targets, vol. 5913. San Diego, CA, USA: SPIE, Aug. 2005, pp. 230-241.

[4] J. W. Koch, "Bayesian approach to extended object and cluster tracking using random matrices," IEEE Transactions on Aerospace and Electronic Systems, vol. 44, no. 3, pp. 1042-1059, Jul. 2008

[5] J. W. Koch and M. Feldmann, "Cluster tracking under kinematical constraints using random matrices," Robotics and Autonomous Systems, vol. 57, no. 3, pp. 296-309, Mar. 2009.

[6] M. Feldmann, D. Fränken, and J. W. Koch, "Tracking of extended objects and group targets using random matrices," IEEE Transactions on Signal Processing, vol. 59, no. 4, pp. 1409-1420, Apr. 2011.

[7] W. Wieneke and J. W. Koch, "Probabilistic tracking of multiple extended targets using random matrices," in Proceedings of SPIE Signal and Data Processing of Small Targets, Orlando, FL, USA, Apr. 2010.

[8] M. Baum and U. D. Hanebeck, "Random hypersurface models for extended object tracking," in IEEE International Symposium on Signal Processing and Information Technology (ISSPIT), Ajman, United Arab Emirates, Dec. 2009, pp. 178-183.

[9] M. Baum, B. Noack, and U. D. Hanebeck, "Extended Object and Group Tracking with Elliptic Random Hypersurface Models," in Proceedings of the International Conference on Information Fusion, Edinburgh, UK, Jul. 2010.

[10] K. Granström, C. Lundquist, and U. Orguner, "Tracking Rectangular and Elliptical Extended Targets Using Laser Measurements," in Proceedings of the International Conference on Information Fusion, Chicago, IL, USA, Jul. 2011, pp. 592-599.

[11] C. Lundquist, K. Granström, and U. Orguner, "Estimating the Shape of Targets with a PHD Filter," in Proceedings of the International Conference on Information Fusion, Chicago, IL, USA, Jul. 2011, pp. 49-56.

[12] M. Baum and U. D. Hanebeck, "Shape Tracking of Extended Objects and Group Targets with Star-Convex RHMs," in Proceedings of the International Conference on Information Fusion, Chicago, IL, USA, Jul. 2011, pp. 338-345.

[13] H. Zhu, C. Han, and C. Li, "An extended target tracking method with random finite set observations," in Proceedings of the International Conference on Information Fusion, Chicago, IL, USA, Jul. 2011, pp. 73-78.

[14] R. Mahler, Statistical Multisource-Multitarget Information Fusion. Norwood, MA, USA: Artech House, 2007.

[15] — "Multitarget Bayes filtering via first-order multi target moments," IEEE Transactions on Aerospace and Electronic Systems, vol. 39, no. 4 , pp. 1152-1178, Oct. 2003.

[16] B.-N. Vo and W.-K. Ma, "The Gaussian mixture probability hypothesis density filter," IEEE Trans. Signal Process., vol. 54, no. 11, pp. 40914104, Nov. 2006.

[17] D. E. Clark and B.-N. Vo, "Convergence analysis of the Gaussian mixture PHD filter,' IEEE Transactions on Signal Processing, vol. 55, no. 4, pp. 1204-1212, Apr. 2007.

[18] B.-N. Vo, S. Singh, and A. Doucet, "Sequential monte carlo methods for multitarget filtering with random finite sets," IEEE Transactions on Aerospace and Electronic Systems, vol. 41, no. 4, pp. 1224-1245, Oct. 2005.

[19] D. E. Clark and J. Bell, "Convergence results for the particle PHD filter," IEEE Transactions on Signal Processing, vol. 54, no. 7, pp. 2652-2661, Jul. 2006.

[20] A. Johansen, S. Singh, A. Doucet, and B.-N. Vo, "Convergence of the SMC implementation of the PHD filter," Methodology and Computing in Applied Probability, vol. 8, no. 2, pp. 265-291, 2006.

[21] R. Mahler, "PHD filters for nonstandard targets, I: Extended targets," in Proceedings of the International Conference on Information Fusion, Seattle, WA, USA, Jul. 2009, pp. 915-921.

[22] K. Granström, C. Lundquist, and U. Orguner, "Extended Target Tracking using a Gaussian Mixture PHD filter," IEEE Transactions on Aerospace and Electronic Systems, vol. 48, no. 4, pp. 3268-3286, Oct. 2012.

[23] K. Granström, C. Lundquist, and U. Orguner, "A Gaussian mixture PHD filter for extended target tracking," in Proceedings of International Conference on Information Fusion, Edinburgh, Scotland, May 2010.

[24] K. Granström and U. Orguner, "A PHD filter for tracking multiple extended targets using random matrices," IEEE Transactions on Signal Processing, vol. 60, no. 11, pp. 5657-5671, Nov. 2012.

[25] A. Swain and D. Clark, "The PHD filter for extended target tracking with estimable shape parameters of varying size," in Proceedings of the International Conference on Information Fusion, Singapore, Jul. 2012.
[26] A. Carmi, F. Septier, and S. J. Godsill, "The Gaussian mixture MCMC particle algorithm for dynamic cluster tracking," Automatica, vol. 48, no. 10, pp. 2454-2467, Oct. 2012.

[27] D. E. Clark and S. J. Godsill, "Group target tracking with the Gaussian mixture probability hypothesis density filter," in International Conference on Intelligent Sensors, Sensor Networks and Information (ISSNIP), Melbourne, Australia, Dec. 2007, pp. 149-154.

[28] B.-T. Vo, B.-N. Vo, and A. Cantoni, "Bayesian filtering with random finite set observations," IEEE Transactions on Signal Processing, vol. 56, no. 4, pp. 1313-1326, Apr. 2008.

[29] O. Erdinc, P. Willett, and Y. Bar-Shalom, "Probability hypothesis density filter for multitarget multisensor tracking," in Proceedings of the International Conference on Information Fusion, Philadelphia, CA, USA, july 2005, pp. 146-153.

[30] R. Mahler, "PHD filters of higher order in target number," IEEE Trans. Aerosp. Electron. Syst., vol. 43, no. 4, pp. 1523-1543, Oct. 2007.

[31] B.-T. Vo, B.-N. Vo, and A. Cantoni, "Analytic implementations of the cardinalized probability hypothesis density filter," IEEE Transactions on Signal Processing, vol. 55, no. 7, pp. 3553-3567, Jul. 2007.

[32] A. Swain and D. Clark, "Extended object filtering using spatial independent cluster processes," in Proceedings of the International Conference on Information Fusion, Edinburgh, UK, Jul. 2010.

[33] F. Lian, C. Han, W. Liu, J. Liu, and J. Sun, "Unified cardinalized probability hypothesis density filters for extended targets and unresolved targets," Signal Processing, vol. 92, no. 7, pp. 1729-1744, 2012.

[34] U. Orguner, C. Lundquist, and K. Granström, "Extended Target Tracking with a Cardinalized Probability Hypothesis Density Filter," in Proceedings of the International Conference on Information Fusion, Chicago, IL, USA, Jul. 2011, pp. 65-72.

[35] U. Orguner, C. Lundquist, and K. Granström, "Extended target tracking with a cardinalized probability hypothesis density filter," Department of Electrical Engineering, Linköping University, SE-581 83 Linköping, Sweden, Tech. Rep. LiTH-ISY-R-2999, Mar. 2011. [Online]. Available: http://www.control.isy.liu.se/research/reports/2011/2999.pdf

[36] A. K. Gupta and D. K. Nagar, Matrix variate distributions, ser. Chapman \& Hall/CRC monographs and surveys in pure and applied mathematics. Chapman \& Hall, 2000.

[37] K. Granström and U. Orguner, "Estimation and Maintenance of Measurement Rates for Multiple Extended Target Tracking," in Proceedings of the International Conference on Information Fusion, Singapore, Jul. 2012.

[38] - "On the Reduction of Gaussian inverse Wishart mixtures," in Proceedings of the International Conference on Information Fusion, Singapore, Jul. 2012.

[39] U. Orguner. (2010, Nov.) CPHD filter derivation for extended targets. ArXiv:1011.1512v2. [Online]. Available: http://arxiv.org/abs/ 1011.1512v2

[40] D. E. Clark, K. Panta, and B.-N. Vo, "The GM-PHD Filter Multiple Target Tracker," in Proceedings of the International Conference on Information Fusion, Florence, Italy, Jul. 2006.

[41] K. Panta, D. Clark, and B.-N. Vo, "Data association and track management for the Gaussian mixture probability hypothesis density filter," IEEE Transactions on Aerospace and Electronic Systems, vol. 45, no. 3, pp. 1003-1016, Jul. 2009.

[42] D. Schuhmacher, B.-T. Vo, and B.-N. Vo, "A consistent metric for performance evaluation of multi-object filters," IEEE Transactions on Signal Processing, vol. 56, no. 8, pp. 3447-3457, Aug. 2008.

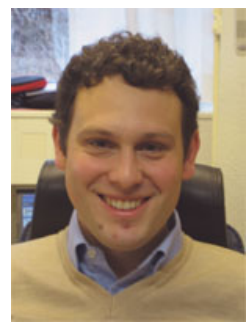

Christian Lundquist received the M.Sc. degree in Automation and Mechatronics Engineering from Chalmers University of Technology, Gothenburg, Sweden, in 2003. He received the Ph.D. degree in 2011, at the Department of Electrical Engineering at Linköping University, Linköping, Sweden, where he now works as a postdoctoral fellow.

Between 2004 and 2007, he worked on active steering systems at ZF Lenksysteme GmbH, Germany. His research interests include sensor fusion and target tracking for automotive applications.

$\mathrm{He}$ is CEO and co-founder of the company SenionLab, developing navigation and positioning solutions for indoor usage. 


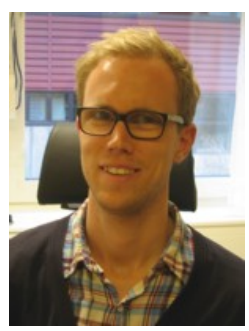

Karl Granström received the M.Sc. degree in Applied Physics and Electrical Engineering in 2008 at Linköping University, Sweden. He received the Ph.D. degree in 2012, at the Department of Electrical Engineering at Linköping University, where he now works as a postdoctoral fellow. His research interests include sensor fusion, target tracking, mapping and localisation, especially for mobile robots.

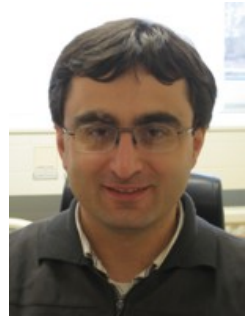

Umut Orguner is an Assistant Professor at Department of Electrical \& Electronics Engineering of Middle East Technical University, Ankara, Turkey. He got his B.S., M.S. and Ph.D. degrees all in Electrical Engineering from the same department in 1999, 2002 and 2006 respectively. He held a postdoctoral position between 2007 and 2009 at the Division of Automatic Control at the Department of Electrical Engineering of Linköping University, Linköping, Sweden. Between 2009 and 2012 he was an Assistant Professor at the Division of Automatic Control at the Department of Electrical Engineering of Linköping University, Linköping, Sweden.

His research interests include estimation theory, multiple-model estimation, target tracking, and information fusion. 